\title{
MObjects-A Novel Method for the Visualization and Interactive Exploration of Defects in Industrial XCT Data
}

\author{
Andreas Reh, Christian Gusenbauer, Johann Kastner, Eduard Gröller, and Christoph Heinzl
}
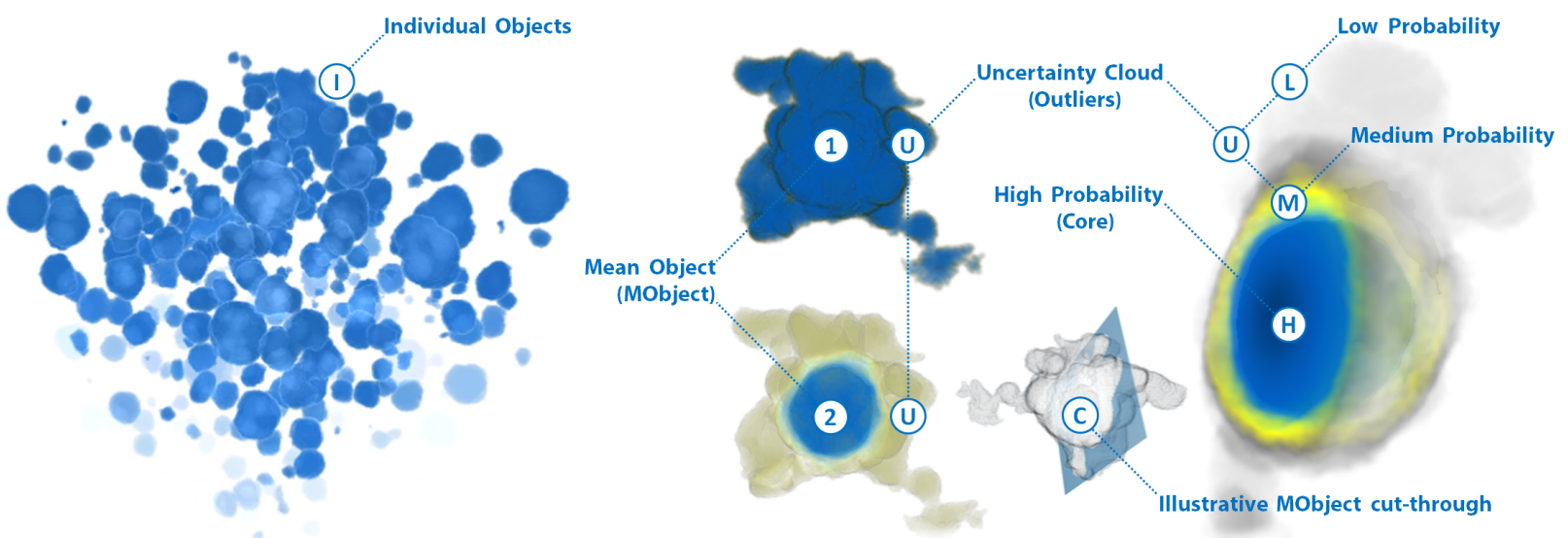

Fig. 1. From a set of individual objects (I) a MObject is calculated. MObjects are visualized by transfer functions based on the probability of each voxel of belonging to the MObject (1 and 2). The MObject cut-through (C) shows areas with high probability $(\mathrm{H})$ in the center. Medium (M) and low (L) probabilities represent the uncertainty cloud (U) showing outliers of individual objects.

\begin{abstract}
This paper describes an advanced visualization method for the analysis of defects in industrial 3D X-Ray Computed Tomography (XCT) data. We present a novel way to explore a high number of individual objects in a dataset, e.g., pores, inclusions, particles, fibers, and cracks demonstrated on the special application area of pore extraction in carbon fiber reinforced polymers (CFRP). After calculating the individual object properties volume, dimensions and shape factors, all objects are clustered into a mean object (MObject). The resulting MObject parameter space can be explored interactively. To do so, we introduce the visualization of mean object sets (MObject Sets) in a radial and a parallel arrangement. Each MObject may be split up into sub-classes by selecting a specific property, e.g., volume or shape factor, and the desired number of classes. Applying this interactive selection iteratively leads to the intended classifications and visualizations of MObjects along the selected analysis path. Hereby the given different scaling factors of the MObjects down the analysis path are visualized through a visual linking approach. Furthermore the representative MObjects are exported as volumetric datasets to serve as input for successive calculations and simulations. In the field of porosity determination in CFRP non-destructive testing practitioners use representative MObjects to improve ultrasonic calibration curves. Representative pores also serve as input for heat conduction simulations in active thermography. For a fast overview of the pore properties in a dataset we propose a local MObjects visualization in combination with a color-coded homogeneity visualization of cells. The advantages of our novel approach are demonstrated using real world CFRP specimens. The results were evaluated through a questionnaire in order to determine the practicality of the MObjects visualization as a supportive tool for domain specialists.
\end{abstract}

Index Terms-3D X-ray computed tomography, carbon fiber reinforced polymers, porosity, parameter space analysis, MObjects

\section{INTROdUCtION AND MOtivation}

Industrial research is continuously increasing efforts in designing newtailored light-weight materials in order to meet the high demands regarding efficiency, environment, safety as well as comfort. Especially

- Andreas Reh is with the University of Applied Sciences Upper Austria, Campus Wels, Austria. E-mail: andreas.reh@fh-wels.at.

- Christian Gusenbauer is with the University of Applied Sciences Upper Austria, Campus Wels, Austria. E-mail: christian.gusenbauer@fh-wels.at.

- Johann Kastner is with the University of Applied Sciences Upper Austria, Campus Wels, Austria. E-mail: johann.kastner@fh-wels.at.

- Eduard Gröller is with the Institute of Computer Graphics and Algorithms, Vienna University of Technology, Austria. E-mail: groeller@cg.tuwien.ac.at.

- Christoph Heinzl is with the University of Applied Sciences Upper Austria, Campus Wels, Austria. E-mail: christoph.heinzl@fh-wels.at.

Manuscript received 31 March 2013; accepted 1 August 2013; posted online 13 October 2013; mailed on 4 October 2013.

For information on obtaining reprints of this article, please send

e-mail to:tvcg@computer.org. in the aeronautics industry a high demand for advanced composite materials is observable. Aircrafts of the future will be made of more than $50 \%$ of these novel composite materials. Carbon fiber reinforced polymers (CFRPs) are currently considered as the most promising candidate since this material is outperforming the majority of conventional materials. As a result of the manufacturing process this material tends to have pores inside [25]. Pores in the material are typically inclusions of air. As they have an impact on the mechanical properties of the component, their determination and evaluation is an important task in quality control and a particular challenge for non-destructive testing (NDT) practitioners. Besides the characterization of individual pores, their spatial distribution in the tested component is a relevant factor. For example, a high concentration of pores in certain regions leads to different material characteristics as compared to a homogenous distribution of the pores.

The current state-of-the-art method for non-destructive porosity determination in aeronautics is ultrasonic testing. Characterization using ultrasonics is required by various aeronautic and automotive standards. The porosity is estimated from ultrasonic velocity and attenuation of sound waves using a calibration curve which is based on the contained 


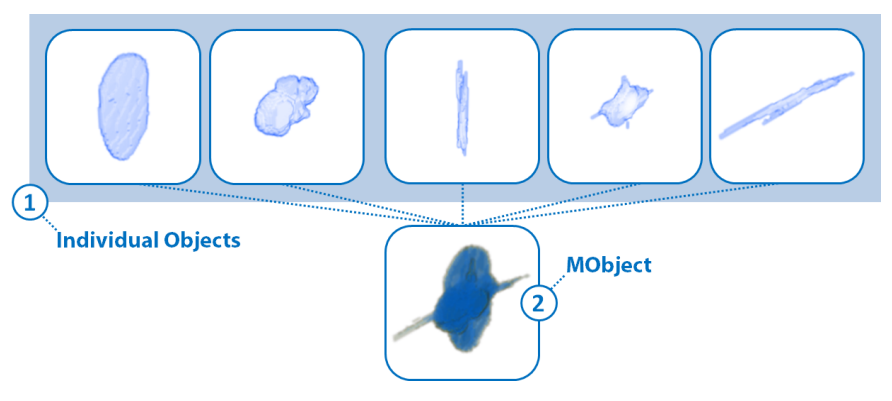

Fig. 2. Illustration of the MObject calculation showing pores of a CFRP dataset. (1) First the individual pores are spatially aligned according to their centers. (2) Second the MObject is calculated by summing up the voxels.

pores. Although the pores and their shape factors are strongly related to the manufacturing process, the same calibration curve is used for components from diverse manufacturing operations. This leads to inaccurate results. Active thermography is a complementary NDT method for porosity determination in CFRP components. For active thermography heat is induced with flashes of light and the propagation of the surface temperature is measured. As the heat propagation depends on the thermal diffusivity of the component, the heat conduction model is fundamental for the accuracy of the generated results [20]. The model is based on simulations, in which the sizes and shape factors of the pores play an important role, although they are not exactly known.

To avoid these drawbacks and to support NDT practitioners, we apply 3D X-Ray Computed Tomography (XCT). It is an NDT method with an increasing importance in the field of aeronautics. With XCT the tested component is placed on a rotary plate between an X-ray source and a detector. For a series of angular positions, 2D projection images are acquired and a 3D volumetric dataset is reconstructed. Due to the high spatial resolution, industrial XCT allows to detect a high number of pores (individual objects) inside. At first glance these pores have similar shapes and it is a difficult and tedious task to evaluate the data by identifying representative structures of interest in the dataset.

As a solution to this problem we introduce mean objects ( $\mathrm{MOb}$ jects). The pores in the 3D dataset are examined individually (see Figure 2 (1)). To compute the MObject, the pores are spatially aligned according to their centers. The value of each voxel in the MObject is the sum of individual pores that overlap it (see Figure 2 (2)). By normalizing the MObject to 1, each voxel holds the probability of belonging to the MObject. Figure 1 illustrates our novel visualization approach showing individual objects (I) and the visualization with an illustrative MObject cut-through (C). A MObject is visualized by transfer functions based on the probability of each voxel in the MObject dataset (1 and 2). Areas with a high probability $(\mathrm{H})$ represent the MObject core in the center. The surrounding medium and low probabilities ( $\mathrm{M}$ and $\mathrm{L})$ represent an uncertainty cloud (U) and correspond to outliers of individual objects. The calculated MObject of the whole dataset is then explored by a decomposition using interactive selection. The resulting set of MObjects is called MObject Set. With the help of the MObject Sets it is now possible to find representative MObjects in the dataset. For our specific application of CFRP analysis representative MObjects are structures of interest in the dataset. For example representative mean pores in a CFRP component are nodular and disc-shaped pores within the epoxy resin as well as long and thin micro pores in the fiber bundles. Although our approach can be used for all kinds of defects in material sciences, e.g., pores, inclusions, particles, fibers and even cracks, we will focus in this work on the evaluation of pores.

\section{TASKS AND CONTRIBUtIONS}

The previously introduced problem descriptions and their demands regarding material sciences lead us to the following tasks for the visualization and exploration of MObjects:
- MObjects Visualization (Task 1): A non-destructive testing (NDT) practitioner analyses visual representations of features or objects of interest inside a CFRP dataset regarding their individual properties, e.g., volume, dimensions, or shape factors. Due to the high number of pores the calculation and visualization of MObjects is the most important task.

- Local MObjects Visualization (Task 2): Besides individual pore properties, the spatial distribution of the pores is an important property of the investigated specimen. Non-destructive testing practitioners need a fast overview of the pore homogeneity. To achieve this goal, the dataset is divided into cells. For each cell a local MObject is calculated and visualized.

- MObjects Exploration (Task 3): Ultrasonic testing practitioners and active thermography experts are searching for MObjects as representatives of the structures of interest in the dataset. The MObjects are needed to improve the ultrasonic calibration curve and the simulations of the thermal diffusivity model. Therefore the MObject of a CFRP dataset is explored interactively.

In this paper we employ XCT to determine and visualize pores in CFRP specimens. MObjects of the segmented pores are calculated and visualized. To accomplish Task 1 - Task 3 visualization methods for the interactive exploration of MObjects are introduced. The main contributions of our work are:

- MObjects Calculation and Visualization: We calculate MObjects by clustering a set of individual pores. As the calculation produces probabilities for each voxel, we visualize an uncertainty cloud using transfer functions.

- Homogeneity Visualization using Local MObjects: For a fast homogeneity overview of the specimen we divide the dataset into regular sub-volumes. For each of these volumes we calculate a local MObject. We extend the homogeneity visualization with a color-coding of the sub-volumes regarding their individual properties.

- Interactive Exploration of MObjects: For the interactive exploration and visualization of pores in a CFRP dataset, we introduce two methods. In a beginners mode the MObjects are arranged in a radial design. All possible combinations of the user-defined properties are calculated and visualized. In the $e x$ pert mode, the MObjects are arranged in parallel. They are constructed interactively from one level to the next in order to allow an in-depth exploration of the dataset. The resulting representative MObjects can be exported as volumetric datasets to serve as input for ultrasonic calibrations and active thermography simulations. Each voxel holds the probability of the MObject membership.

\section{Related Work}

In our previous work [23] we introduced a drill-down approach to explore pores in a CFRP dataset. The main focus of this work was the determination of the quantitative porosity and the comparison with existing reference methods like ultrasonic testing, active thermography, and acid digestion. A fast porosity overview with the porosity maps visualization and an individual pore visualization with parallel coordinates was presented and showed satisfying results. However porosity in CFRP is still an interesting and important topic of research. Due to the possible high number of pores in the datasets, occlusions of pores may lead to evaluation problems using an individual pore visualization. We found out that analyzing the pores individually is insufficient in certain evaluation scenarios, e.g., if the mean characteristics of the pores are needed. In this paper, we developed the new MObjects approach which gives the user a fast visual overview of the average pores in the dataset. In the following sections we review the related work for our MObjects visualization and exploration pipeline. 


\subsection{Segmentation}

The segmentation of the individual pores is an essential task. A survey on thresholding techniques by Sezgin and Sankur [24] shows the wide range of approaches and applications. All of these methods assign an object membership based on a density threshold, where no neighborhood information is taken into account. As we showed in our previous work [23] Otsu's thresholding technique [21] leads to satisfying results for industrial XCT data of CFRP specimens. It is an automatic threshold-selection method for bimodal histograms. The histogram is divided into two classes minimizing the intra-class variance and maximizing the inter-class variance. Thus the separability of the resulting classes in gray levels is maximized. As segmentation techniques are exchangeable in the presented workflow and not considered as core of this work, this area is not considered in more detail.

\subsection{Parameter Space Analysis}

The resulting set of segmented pores together with their properties like the volume, dimensions, and shape factors make up a new parameter space, which has to be explored (MObjects Exploration - Task 3). A broad range of methods and applications for parameter space analysis exists. Design galleries by Marks et al. [19] present an interface with an automatically generated selection of different graphics or animations. These can be produced by varying the input parameters, e.g., opacity and color transfer functions for volume rendering. Ma [17] introduces Image Graphs, where the nodes in the graphs show result images. Each edge depicts the change of the rendering parameters between its connected nodes. Changes in the rendering parameters propagate through the graph. Bruckner and Möller [7] developed a system to assist graphics artists in generating special effects, e.g., smoke or explosions. Their visual exploration approach of the parameter spaces allows the user to find the appropriate parameters for the desired results. Torsney-Weir et al. [26] introduced Tuner, a system for parameter finding in image segmentation. Tuner systematically explores the parameter space in two stages. After sampling the parameter space, a statistical model for the estimation of the segmentation algorithm's response is applied. Based on this information the user can navigate through the parameter space to find areas with high response values. Amirkhanov et al. [2] presented a tool for the visual optimality and stability analysis of 3DCT specimen placements. For parameter space analysis a stability widget based on penetration-length calculation, radon-space analysis as well as placement-stability analysis is used. Another approach by Bruckner et al. [8] is realized in the BrainGazer software. The system is used for the exploration and analysis of neural circuits. Visual queries based on semantic and spatial relationships are applied to a database of fruit-fly brains. Berger et al. [5] presented an interactive approach to analyze a sampled parameter space. Other interesting papers concerning our work are available as well [28], [27] and [3].

\subsection{Clustering}

By calculating MObjects, we identify and group similar individual objects with respect to one of their properties. Therefore a cluster algorithm is used. Xu published a detailed survey on clustering algorithms [29]. We let the user decide on the number of clusters, e.g., a norm specifies the number of partitions which have to be made in an evaluation scenario. As the clustering algorithm is easily replaceable, simple k-means is taken for our approach of clustering individual objects. Clustering itself is considered to be out of scope for this work and is not discussed in more detail.

\subsection{Hierarchical Visualization}

The calculation of MObjects is based on the composition and decomposition of mean and individual objects. This leads us to hierarchical visualizations for the interactive exploration of the MObjects (MObjects Exploration - Task 3). A combination of hierarchy visualization and scientific visualization was presented by Balabanian et al. [4]. Their method integrates visualizations for hierarchically organized volumetric datasets. A graph shows the hierarchy and the nodes display the corresponding 3D volumetric data. Brambilla et al. [6] introduced a hierarchical splitting scheme for the analysis of integral surfaces. At each hierarchy level the cuts are chosen according to a surface complexity metric. Ip et al. [13] partition the histogram of a volumetric dataset into an exploration hierarchy using a normalizedcut multilevel segmentation approach. Inspired by these techniques, we introduce our MObject Set visualization. After a decomposition of the dataset's MObject, the user is able to explore the data in a graph, where the nodes show 3D representations of the MObjects.

\subsection{Uncertainty Visualization}

We calculate a new volumetric MObject dataset by aggregating individual objects of one cluster. Each voxel holds the probability of belonging to the newly generated MObject. These probabilities as well as the regions with elevated uncertainty are visualized (MObjects Visualization - Task 1). Kniss et al. [14] present an approach for the interactive exploration of uncertainty including a risk and decision analysis. They render the results of the risk analysis into a unified probabilistic data space. As MObjects are aggregated from all considered individual objects, the approach by Kniss et al. [14] is not suitable for our visualization. Point-based probabilistic surfaces were introduced by Grigoryan and Rheingans [10]. They visualize surfaces with uncertainties using points as display primitives. Although their approach is useful for visualizing uncertainty on surfaces, we can not apply it to our data. Fout and Ma [9] present fuzzy volume rendering. By computing the posteriori uncertainty they provide a verifiable volume rendering. Heinzl et al. [11] compute a probability volume of multimaterial components using a statistical analysis. Similarly we provide insight into the MObjects' data to show the core and the outliers. We also use transfer functions emphasizing a user-defined border of probability. They are suitable for visualizing the a priori-based uncertainty of an MObject.

\subsection{Comparative Visualization}

The issue of visualizing the pore homogeneity in the dataset (Local MObjects Visualization - Task 2) leads us to comparative visualization. Malik et al. [18] refer to a wide range of comparative visualization approaches. Additionally they propose a base tile pattern for a multiimage view for comparative visualization, which is an extension of the checkerboard-pattern approach and attribute blocks. Malik et al. compare 2D slices of volumetric datasets with different measurement parameters. Ahrens et al. [1] visualize differences between scientific simulations. These methods are not applicable to our segmented pores data. We divide the dataset into cells and calculate local MObjects for each cell. Furthermore we add a homogeneity visualization, where we color-code the cells based on the deviation of the local average from the global average.

\subsection{Visualization of Multivariate Data}

Parallel coordinates introduced by Inselberg [12] are a common visualization approach for displaying and filtering multivariate data. Kosara et al. [15] extended parallel coordinates and presented parallel sets for dealing with categorical data. Instead of individual data points, they show data frequencies between the axes and visualize the relations between categories. StratomeX by Lex et al. [16] was inspired by parallel sets, where datasets of genomic data are represented as columns and subtypes as bricks in the columns. Our MObject Set visualization in parallel alignment was inspired by these techniques (MObjects Exploration - Task 3). Parallel coordinates can serve as classifiers for the selection of individual pores and parallel sets share the idea of clustered objects. Due to the usage of categories, both approaches are not applicable to volumetric datasets. They do not visualize the spatial relationship between as well as the appearance of the objects.

\section{MObjects Visualization and Exploration Pipeline}

In our work we want to identify representative structures of interest in an XCT dataset, e.g., pores in a CFRP component. We propose a pipeline for the visualization and interactive exploration of MObjects which is illustrated in Figure 3. In the data acquisition stage (1) 3D XRay Computed Tomography (XCT) data is generated (see Section 4.1). On the resulting volumetric dataset pre-processing steps (2), described 


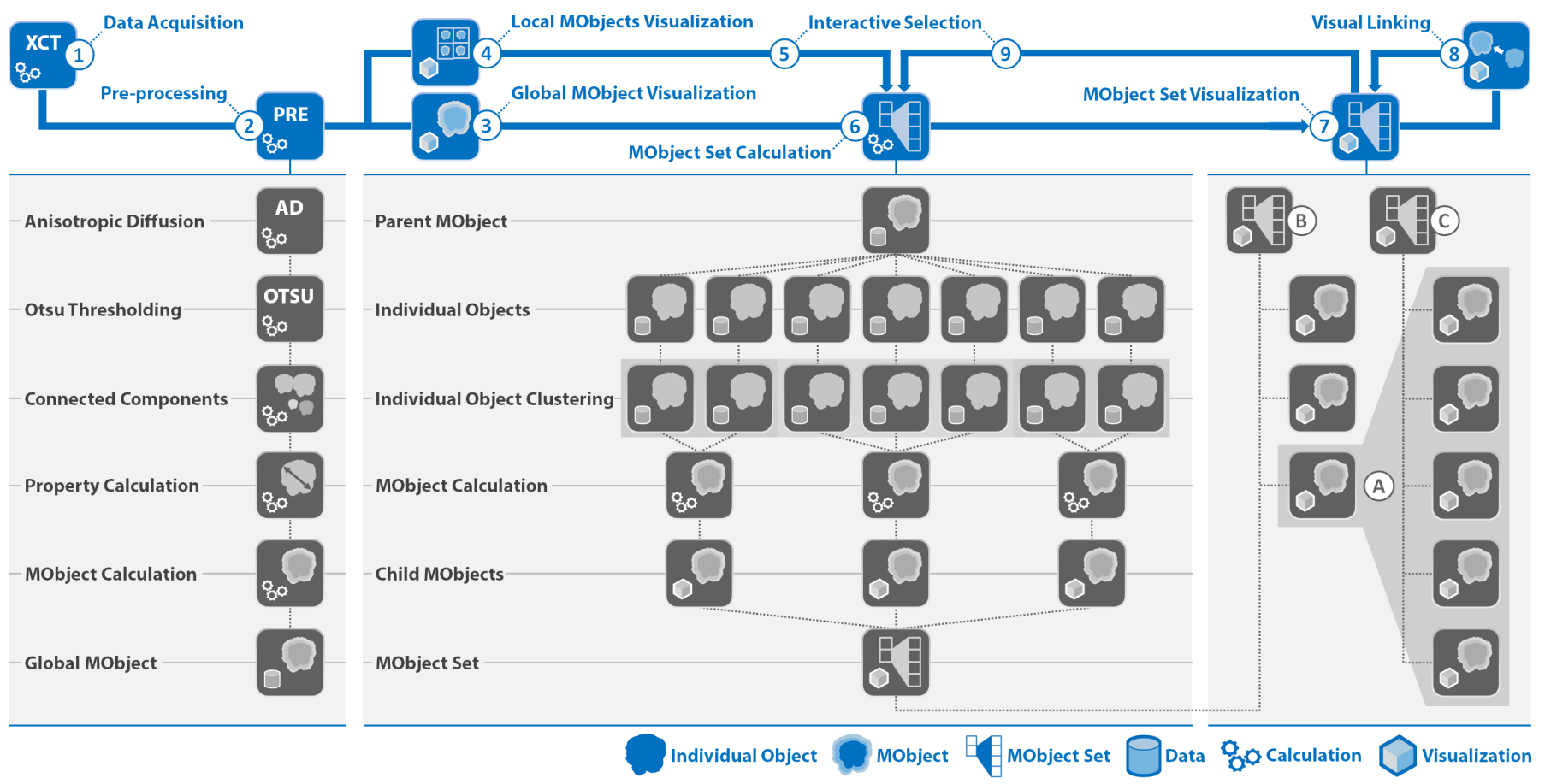

Fig. 3. Pipeline for the visualization and interactive exploration of MObjects in a dataset. The main pipeline is colored in blue, whereas the detailed steps are shown in gray.

in Section 4.2, are performed to calculate the resulting global MObject of the dataset. This MObject is considered as mean pore and represents the aggregation of all pores in the component. For further visualization of the MObject we propose two methods. In our first approach, the global MObjects visualization (3), the global MObject serves as input for further exploration. For a fast overview of the dataset we suggest a local MObjects visualization (4). The dataset is divided into cells and for each cell a local MObject is calculated and shown in combination with a color-coded homogeneity visualization. Through interactive selection (5) the subsequent exploration starts with one of the local MObjects instead of the global one.

All the individual objects in the MObject of interest are then clustered in the MObject Set calculation (6). The parent MObject is further subdivided into new child MObjects based on one of their calculated properties. For example the parent MObject is classified into two child MObjects based on the shape factors of the pores. The first MObject only contains nodular pores, whereas the second one consists of long and thin pores. The user is then able to interact with the resulting MObject Set visualization (7). Through visual linking (8) or interactive selection (9) an iterative calculation and visualization of the $\mathrm{MOb}$ ject Sets will be triggered upon each major change in the classification. By selecting a MObject (A) in a MObject Set (B) a new MObject Set (C) consisting of new child MObjects can be calculated from the selected parent MObject (A).

\subsection{Data Acquisition}

In this work, we use carbon fiber reinforced polymers (CFRP) specimens made of preimpregnated carbon fibers and epoxy resin. For the investigations plates with a size of $17 \times 20 \times 1 \mathrm{~mm}^{3}$ were used. The voxel size of the datasets was $10.5 \mu \mathrm{m}$. The XCT scans were performed on a GE Phoenix|xray nanotom XCT system with a $180 \mathrm{kV}$ nano focus tube. A tube voltage of $60 \mathrm{kV}$, a measurement current of $320 \mu \mathrm{A}$, and $500 \mathrm{~ms}$ integration time at the detector were used as scan parameters. Over-segmentation in the middle of the specimens may occur due to gray value modifications caused by beam hardening during the measurement. Therefore a beam hardening correction was applied during the reconstruction.

\subsection{Pre-processing}

Due to ambient noise in the scanned datasets, we apply anisotropic diffusion as described by Perona and Malik [22] for filtering the data. Anisotropic diffusion smoothes homogenous regions while preserving the edges. We use five iterations with a conductance of one and a time step of 0.0625 seconds. The segmentation of pores is done with the automatic threshold selection method for bimodal histograms by Otsu [21]. For the individual property calculation the objects are labeled after the segmentation by a connected-components filter using 26-connectivity neighborhoods. With the labeled objects we proceed through an individual property calculation stage which is the basis for our MObjects determination. A pore consists of a set of voxels within a regular volumetric grid. To enable interactive exploration and classification of objects the following properties for each individual pore are calculated:

- Pore volume $V_{i}$ : The volume $V_{i}$ of a pore $i$ is the sum of all voxels in the dataset with the same label.

- Dimensions $a_{i}, b_{i}, c_{i}$ : To calculate the extent of the pore along the $\mathrm{x}, \mathrm{y}$ and $\mathrm{z}$ coordinate axes we perform the calculation on the discretized pore. For the extent along the $\mathrm{x}$ axis we determine the two voxels with minimal and maximal $x$ coordinate values respectively. The extents $a_{i}, b_{i}, c_{i}$ are then the differences between the maximal and minimal coordinates along the $\mathrm{x}, \mathrm{y}$ and $\mathrm{z}$ axes of pore $i$.

- Shape factor $F_{i}$ : The shape factor $F_{i}$ of a pore $i$ is defined as the ratio between the pore surface $S_{i}$ and the pore volume $V_{i}$.

- Directional shape factors $F_{(x / z) i}, F_{(y / z) i}, F_{(x / y)_{i}}$ : Mayr et al. [20] define the shape factors of typical ellipsoidal pores for each direction in space as follows: $F_{(x / z)_{i}}=a_{i} / c_{i}, F_{(y / z)_{i}}=b_{i} / c_{i}$ and $F_{(x / y)_{i}}=a_{i} / b_{i}$.

On the basis of these properties a MObject of the whole dataset is calculated in order to explore and visualize representative MObjects in a dataset. 


\section{Visualization}

We introduce novel methods for the visualization and interactive exploration of MObjects which are based on a set of individual objects in a dataset. Besides the calculation and visualization of MObjects (see Section 5.1) we propose a homogeneity visualization of the dataset. It uses local MObjects and a color-coding of the grid-based sub-volumes. The approach is based on the deviation of average individual properties, e.g., the average pore volume in a cell (see Section 5.2). To explore MObjects we present two visualization approaches in Section 5.3 which use MObject Sets in radial and parallel arrangement. Furthermore we discuss interaction techniques for these two methods including visual linking.

\subsection{MObject Visualization}

For the visualization of a single MObject we introduce an uncertainty cloud surrounding the MObject core (see Section 5.1.1). We used transfer functions which are described in more detail in Section 5.1.2. To calculate a MObject all individual objects are spatially aligned by their centers. The center position can be calculated from the dimensions $a_{i}, b_{i}, c_{i}$ of the individual objects or the barycenter of each individual object can be used. Both methods showed similar results for pores in CFRP datasets as pores typically have nodular, disc, or long and elongated shapes. In order to avoid additional calculations, we decided to use the dimension-based approach. When transferring the MObjects idea to other application areas, the centroid calculation has to be reconsidered according to the specific requirements. Although it is possible to rotate and register all individual objects in the calculation, we align them with their original orientation. This transformation without a rotation is important in the specific application area of CFRP analysis which we address in this work. Due to the layer structure of the material the MObject should clearly visualize the different orientations of the individual objects. For each voxel in the individual object dataset, the distances $\Delta x, \Delta y$ and $\Delta z$ to the center are calculated. After the distance calculation for one voxel, the corresponding voxel in the MObject with the same distances $\Delta x, \Delta y$ and $\Delta z$ to the center of the MObject is incremented. This step is done for each voxel of each individual object. To get the corresponding probabilities the resulting MObject dataset is normalized to 1 .

\subsubsection{Uncertainty Cloud}

A MObject is a volumetric dataset, where each voxel holds the probability of belonging to the MObject. This information has to be visualized accordingly. We visualize an uncertainty cloud surrounding the MObject's core. Uncertainty cloud and core are based on the stored probabilities. A high probability at a specific position means

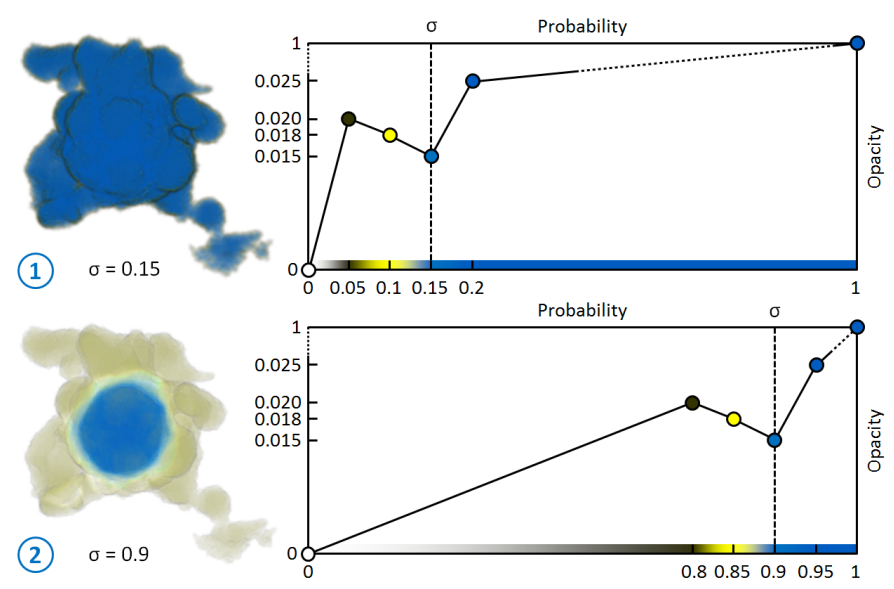

Fig. 4. (1) MObject showing a small uncertainty cloud using a low uncertainty filter $\sigma=0.15$. (2) A high uncertainty filter $\sigma=0.9$ leads to an uncertainty cloud showing the MObject's core and outliers.

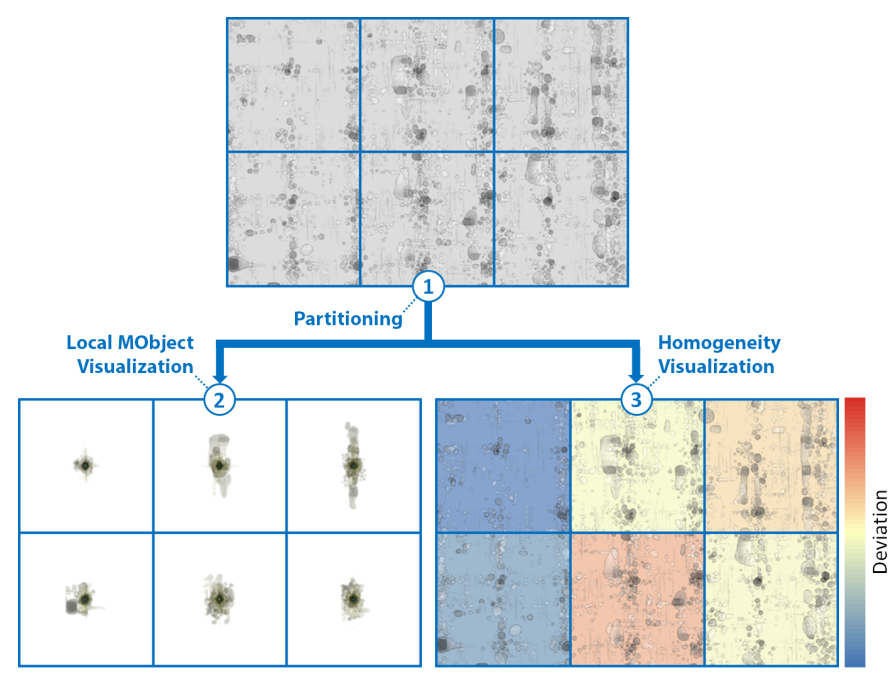

Fig. 5. Based on the partitioning (1) of the volume into cells, local MObjects (2) and a color-coded homogeneity visualization (3) is shown to the user. The color-coding illustrates the deviation of the average cell properties from the global average property. Red indicates the highest positive deviation, whereas blue shows the highest negative deviation.

that nearly all of the individual objects which were summed up to the MObject, include this location. In Figure 1 the uncertainty cloud (U) is illustrated. The probability in the core of the MObject is typically high $(\mathrm{H})$. As the contribution of outliers to the MObject is low, they have a medium (M) or low (L) probability of belonging to the MObject.

\subsubsection{Transfer Function Design}

The uncertainty cloud is visualized using transfer functions. We apply colors for high probabilities (blue) to medium (yellow) and low probabilities (gray). Yellow-grey thus shows the outliers (see Figure 4). The step between high (blue) and medium (yellow) probabilities shows the membership of belonging either to the core or the outliers of the MObject. For the interactive visualization and adaptability to different applications, the user steers this visualization by an uncertainty filter $\sigma$. Figure 4 shows how the gray-yellow-blue gradient is shifted in the transfer function based on $\sigma$. Setting a low uncertainty filter, leads to a small uncertainty cloud and a visualization strongly representing the surface of the MObject (1). In the case of using a high uncertainty filter, one can clearly determine the core of the MObject and see the surrounding outliers (2). To emphasize the uncertainty cloud, we apply a lower opacity value at the position of $\sigma$ in the transfer function.

\subsection{Local MObjects Visualization}

Pore homogeneity is an important criterion in material sciences. We already covered this topic with our porosity maps visualizing the porosity in a CFRP specimen [23]. As the homogeneity of the different pore properties is also helpful to find interesting regions for a further detailed analysis, this information has to be visualized in an easy to understand and intuitive way. We propose a homogeneity visualization using local MObjects and a comparative homogeneity visualization with color-coded cells. Figure 5 describes our approach in more detail. First the volumetric dataset is partitioned into subvolumes (1). We refer to them as cells. The cell size depends on the biggest pore extents in the dataset as it should fit in a cell without overlapping. Based on this partitioning we implement two visualizations to convey homogeneity to the user. In our first method we visualize local MObjects (2). For each cell a MObject is calculated and centered in the cell. All pores whose centers are inside the cell are considered. The same transfer function is used for all local MObjects.

In our second approach the average pore properties and their deviations to the global average properties of the whole dataset are calcu- 


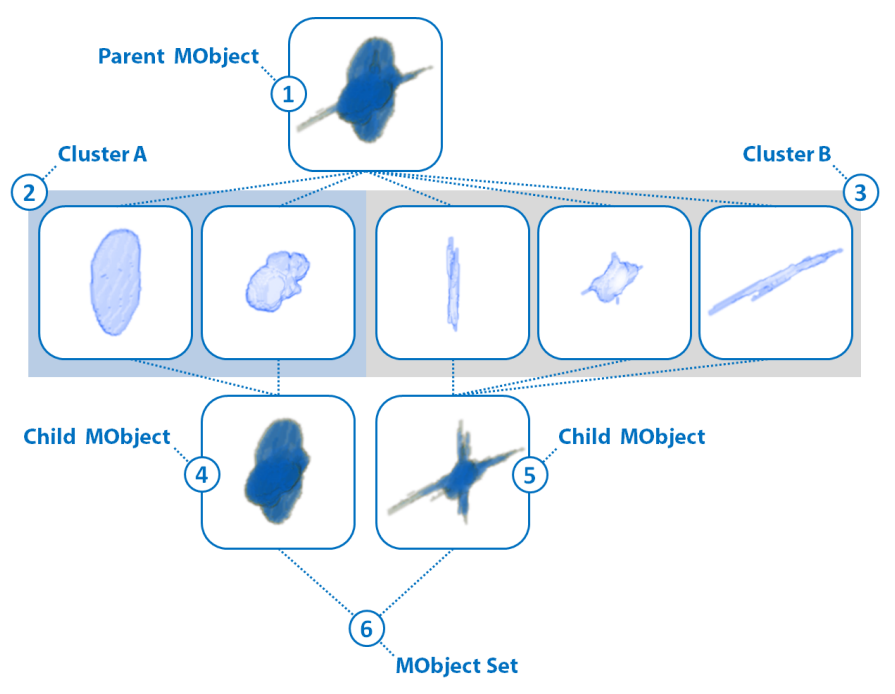

Fig. 6. Illustration of the MObject Set calculation showing pores of a CFRP dataset. All individual pores of the parent MObject (1) are divided into two clusters A (2) and B (3). The resulting child MObjects (4 and 5) make up a new MObject Set (6).

lated. The user is able to guide this comparative homogeneity visualization by selecting a property. Each cell is colored according to its deviation from the selected global property (3). For color-coding the cells we apply a diverging colormap from blue over yellow to red to show the negative and positive deviation of the average cell properties from the global average property of the specimen.

\subsection{MObject Set Visualization}

For the interactive exploration of MObjects in a dataset we provide a visual representation to the user. A parent MObject can be divided into a set of child MObjects which together make up a new MObject Set. All individual objects which belong to the parent MObject are clustered based on one of the calculated properties. For each cluster a new child MObject is calculated. The child MObjects together form a new MObject Set. The MObject Set may be explored interactively with a recursive selection and decomposition of the included child $\mathrm{MOb}$ jects. Figure 6 illustrates this process using individual pores of a CFRP dataset. The parent MObject (1) is split up into the contained individual objects. First, these individual objects are clustered according to their shape factors into two clusters A (2) and B (3). Second, all in- dividual objects in a cluster are aggregated to new child MObjects (4 and 5). These child MObjects make up a new MObject Set (6). For the clustering we provide two possibilities to the user:

- Automatic Clustering: The automatic clustering mode uses kmeans clustering to assign the individual objects to classes. All the individual objects are classified into $k$ clusters where each individual object belongs to the cluster with the closest mean. The user decides on the number $k$ of clusters and which object property should be used for clustering.

- User-defined Clustering: If the user wants to select the cluster centers by himself, it is possible to override the automatic clustering. One reason may be that specific domain expert knowledge from previous analyses or a norm specifies the clustering for a certain property. In this case the user is able to modify the clustering done by the automatic approach using a simple dialog.

After clustering according to a certain property, a child MObject is calculated for each cluster out of all individual objects that belong to it. For the MObject Set visualization we propose then two modes. Section 5.3.1 describes the beginners mode where we introduce the radial MObject Set visualization. The expert mode with the parallel MObject Set visualization is described in Section 5.3.2.

\subsubsection{Radial MObject Set Visualization (Beginners Mode)}

For inexperienced users, setting the number of classes for clustering and specifying the sequence in which the properties shall be explored can be a challenging task. To give a better overview of the data and the resulting MObjects we provide a beginners mode for an interactive exploration of MObjects. Figure 7 (A) illustrates the MObject Set visualization in a radial arrangement. In most evaluation scenarios two properties are sufficient (e.g., pore volume and shape factor). The user selects the number of classes and the desired properties for the MObject Set calculations. The radial design allows to render all possible combinations between the properties (A-1) and (A-2) and not only one at a time (see parallel MObject Set visualization in Section 5.3.2). Based on this visualization, parameters for the expert mode can be found more easily. It further reduces the error rate of selecting undesired MObjects while exploring the data. Through interactive selection (A-3) the user is able to switch to the expert mode. There the MObject Sets (A-4) and (A-5) are displayed in parallel arrangement, according to the previously selected path.

\subsubsection{Parallel MObject Set Visualization (Expert Mode)}

The before described radial MObject Set visualization (see Section 5.3.1) leads to a fixed classification of the individual objects. Experienced users want to cluster the pores based on their properties in a

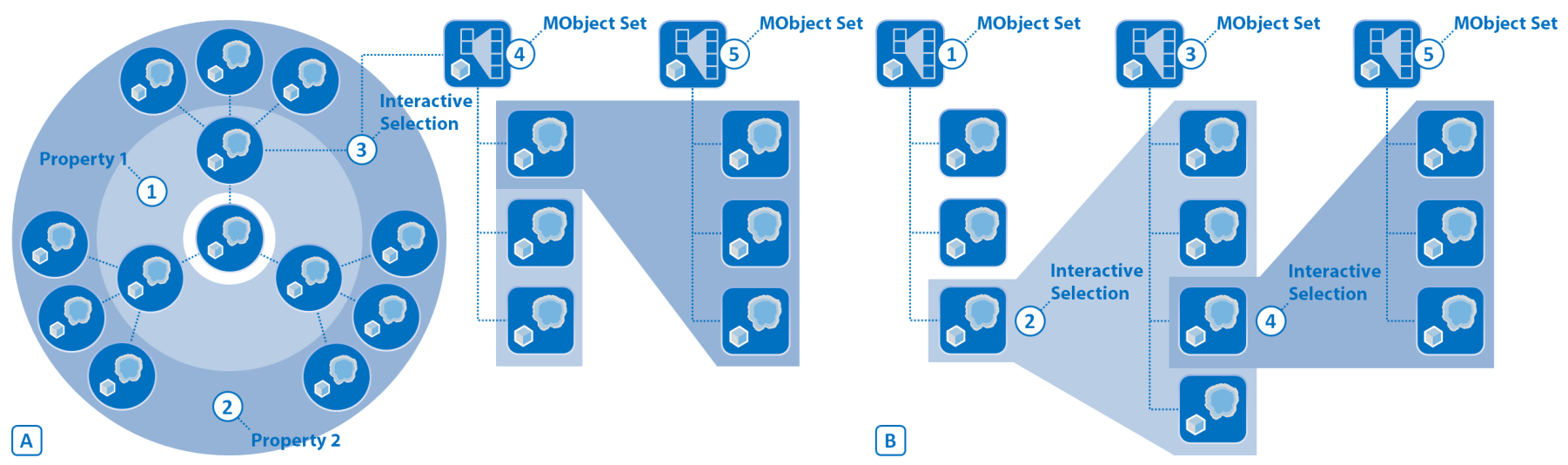

Fig. 7. (A) The radial MObject Set visualization shows all possible combinations between the properties (A-1 and A-2). (A-3) The user is able to switch to parallel alignment which shows MObject Sets (A-4 and A-5) along the selected path. (B) Parallel MObject Set visualization for a step by step exploration. MObject Sets (B-1, B-3 and B-5) are calculated and visualized after interactive selections (B-2 and B-4). 
sequence that best fits their needs to extract the MObjects of interest. This sequence of properties may be different from case to case. To support this situation we present the parallel MObject Set visualization which is illustrated in Figure 7 (B). MObject Sets are calculated and visualized after interactive selections. All individual objects of the dataset are clustered based on a property and a user-defined number of clusters, e.g., three shape factor classes. The resulting MObjects are shown in a MObject Set (B-1). Through interactive selection of a MObject (B-2), the user is able to iteratively repeat the before described step of MObject Set calculation to explore the selected MObject. As a result a new child MObject Set (B-3) is calculated and visualized. As one would commonly use different properties as cluster criteria, it is possible to repeat the interactive selection step (B-4) to calculate another MObject Set (B-5) and finally explore the whole dataset to find the desired MObjects.

\subsubsection{Scaling through Visual Linking}

In our visualization all MObjects are rendered in individual frames of the same size. The MObjects are scaled to best fit their corresponding rendering frame. Our visual linking approach connects all MObjects along the selection path so that it becomes obvious where a child MObject is located inside the parent MObject. Furthermore the scaling of a considered MObject in the hierarchical relationship is represented. To do so, the transfer function for all MObjects except the selected MObject in the visualization is changed in a way, that only the surfaces of the MObjects are shown in grey with a high opacity. The selected MObject is then shown in all MObjects along the selection path. Figure 8 illustrates this approach using a parallel MObject Set visualization with two MObject Sets (A) and (B). When selecting a child MObject (1) visual linking shows the selected MObject inside the parent MObject (2) of the parent MObject Set (B).

\section{Results and Evaluation}

The initial design of the MObjects visualization is motivated by a high demand of material characterizations in the aeronautics industry. Especially carbon fiber reinforced polymers (CFRP) show a great potential because of their increased stiffness and strength-to-weight ratio. As a result of the manufacturing process, CFRP tends to have pores inside. They can occur as nodular pores or crack-shaped delaminations in the epoxy resin or as long and thin micro pores inside the fiber bundles. In the following sections we show the local MObjects visualization including the color-coded homogeneity visualization (see Section 6.1) as well as the radial (see Section 6.2) and parallel (see Section 6.3) MObject Set visualization to explore a high number of individual pores. For the further evaluation we gathered user feedback from domain specialists through a questionnaire (see Section 6.4). The results show a CFRP dataset with a size of $1800 \times 1600 \times 100$ voxel and a porosity of $1.94 \%$. The computational time including all preprocessing stages, the calculation of the global MObject and the local

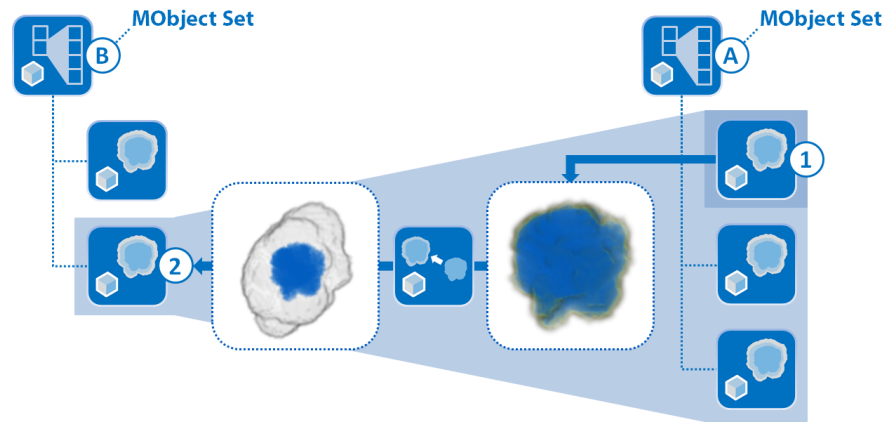

Fig. 8. Illustration of the visual linking approach. After selecting a MObject (1) in a MObject Set (A), visual linking shows the selected child MObject inside the parent MObject (2) of the parent MObject Set (B).

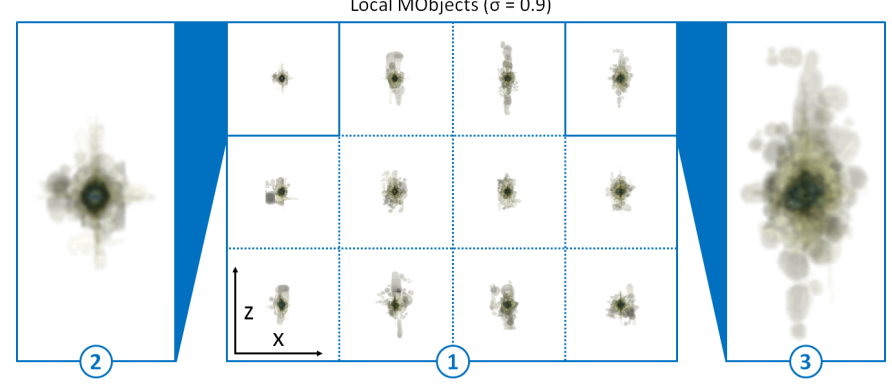

Fig. 9. (1) Local MObjects visualization using a high uncertainty filter $\sigma=0.9$. (2 and 3) Enlarged visualization of two MObjects.

MObjects as well as the cell calculations for the color-coded homogeneity visualization was about 17 minutes on an Intel Xeon X5680 workstation with 48 GB RAM. We have integrated the pipeline in our framework iAnalyse. The MObject calculation and visualization stages are still non-optimized prototypes. As these prototypes are suitable to test the acceptability of our approach using real-world components, the major focus was not the optimization at this stage of the development. With reduced calculation times due to optimization we expect to be in the range of 1 minute and below which is acceptable in this application area.

\subsection{Local MObjects Visualization}

In our local MObjects visualization the CFRP dataset is partitioned into a $4 \times 3$ grid of cells. The number of rows and columns in the grid is calculated automatically depending on the biggest pore extents in the dataset. We ensure that the MObjects fit into the cells without overlapping. For each cell a local MObject is calculated and shown to the user (see Figure 9 (1)). The MObjects can be visualized using different uncertainty filters, e.g., $\sigma=0.9$. Figure 9 ( 2 and 3 ) shows two enlarged MObjects from (1). As the MObjects' cores look similar, there is a homogenous distribution of small individual pores over the dataset in $\mathrm{x}$ direction, whereas big individual pores can be seen as outliers in (3). These outliers lead to an inhomogeneous porosity distribution in the specimen. Based on the given partitioning the user can switch to the comparative homogeneity visualization with colorcoded cells as shown in Figure 10. Without coloring the cells (1) it is
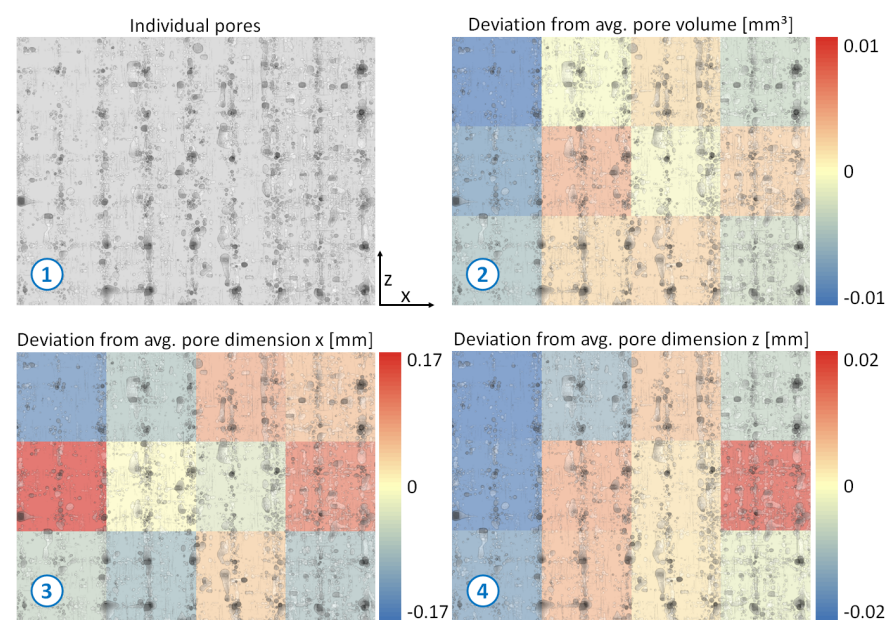

Fig. 10. (1) Visualization of the CFRP dataset. Homogeneity visualization of the deviation from the average pore (2) volume, (3) dimension $\mathrm{x}$, and (4) dimension $\mathrm{z}$ in a $4 \times 3$ grid showing the CFRP dataset in the background. 


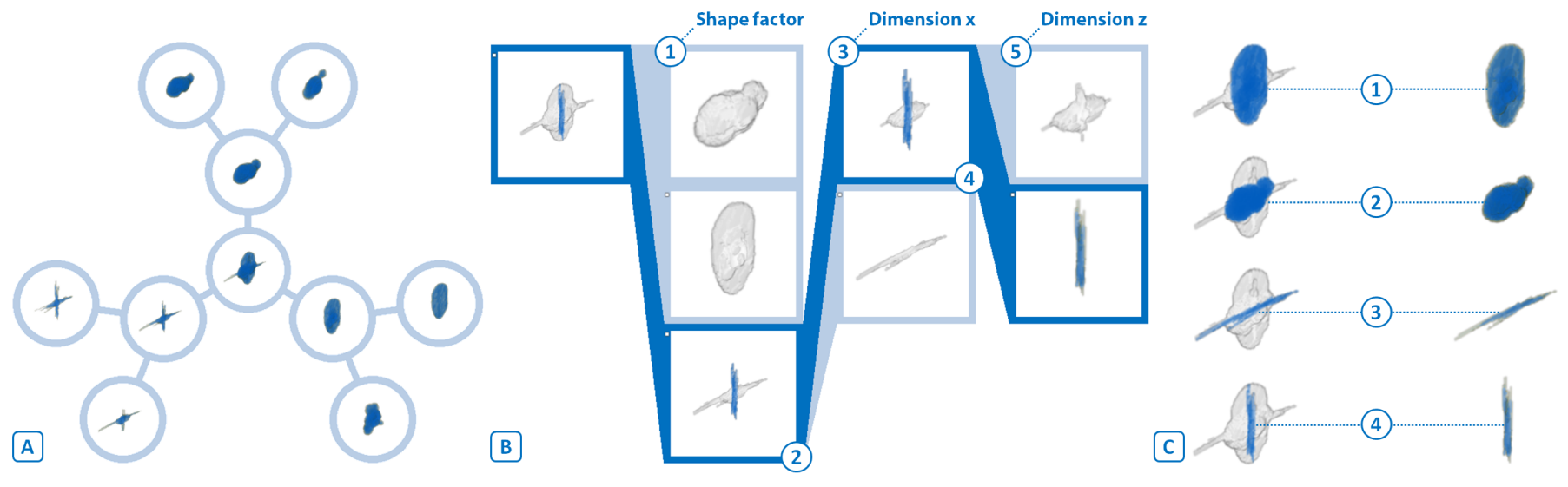

Fig. 11. (A) Radial MObject Set visualization of a CFRP dataset showing combinations of the properties shape factor and volume. (B) Parallel MObject Set visualization of a CFRP dataset showing the selected path and visual linking. (C) Representative mean pores of a CFRP dataset showing the visual linking visualization (left column) and the MObjects (right column). (C-1 and C-2) Nodular and disc-shaped pores. (C-3 and C-4) Long and thin micro pores in $\mathrm{x}$ and $\mathrm{z}$ direction.

hard to gain information about the pore homogeneity. By applying the color-coding, the user is able to see the homogeneity of one property. Our visualization shows the deviation of the average pore volume (2), dimension $\mathrm{x}(3)$, and dimension $\mathrm{z}(4)$ from the corresponding average global property. Further properties we can show are the shape factor and the dimension in y direction. Blue colors show a high negative deviation whereas red indicates a high positive deviation. In this example the homogeneity regarding the pore volume is particularly interesting. A trend can be seen, where in the left part of the specimen the average pore volume is significantly lower (2). Related to active thermography calculations, the homogeneity of the pore dimensions in $\mathrm{x}$ and $\mathrm{z}$ direction is of interest. Especially the homogeneity of the dimension in $x$ direction (3) shows cells with a high deviation with respect to each other. For the homogeneity of the dimension in $\mathrm{z}$ direction (4) lower values in the left part of the specimen are visualized similar to the pore volume deviation.

\subsection{Radial MObject Set Visualization}

In the beginners mode the MObject Sets are arranged in a radial layout. The user can select the desired properties and the number of classes for the MObject Set calculations. The resulting visualization allows the user to see all possible combinations between the properties to get a better overview of the data and the resulting MObjects. Figure 11 (A) shows the radial MObject Set visualization for a CFRP dataset. The MObject of the whole dataset is shown in the center of the radial arrangement. The inner circle shows the MObject classifications based on three shape factor sub-classes. Each of these sub-classes is split up into two sub-classes according to pore volume. Hence all possible combinations of pore shape factor and volume are visualized in one view. Based on this information the user is able to draw conclusions about the individual pores. Using this detailed pore overview, it is easier to parameterize the expert mode (see Section 6.3).

\subsection{Parallel MObject Set Visualization}

The parallel MObject Set visualization shows MObject Sets in a parallel arrangement. The user can interactively explore the MObjects. The results of a CFRP dataset are depicted in Figure 11 (B) using visual linking. First the parent MObject is split into three user-defined shape factor classes (B-1). The three MObjects make up a new MObject Set. After the selection of the last MObject in this set (B-2), it is split into two classes based on the dimension in $\mathrm{x}$ direction (B-3). This step allows to separate the cross-shaped long and thin micro pores. Finally, the first class of the new MObject Set (B-4) is split into two classes based on the dimension in $\mathrm{z}$ direction (B-5). We used the parallel MObject Set visualization to find the representative mean pores in the CFRP dataset (see Figure 11 (C)). As a result of the manufacturing process nodular and disc-shaped pores propagate in the epoxy resin (C-1) and (C-2). Long and thin micro pores occur in $\mathrm{x}$ and $\mathrm{z}$ direction due to the twill-weave pattern of the fiber layers (C-3) and (C-4).

\subsection{Evaluation Questionnaire}

For the evaluation, a questionnaire (see Table 1) was answered by 12 respondents of which make up two groups. The first group consists of domain experts of a company manufacturing aircraft components, including non-destructive testing (NDT) practitioners and various engineers of the CFRP production process. They use our visualizations for gaining new insights into their components in order to draw conclusions about the manufacturing process (MObjects Visualization - Task 1). Furthermore the domain experts use the MObject Set visualization to explore the pores. The resulting representative mean pores are used to improve ultrasonic calibration (MObjects Exploration - Task 3).

The second group of respondents consists of active thermography domain experts. They have a strong demand for a better understanding of the different pores in a CFRP component. Currently geometric primitives are used for mathematical simulations of a heat conduction model. Based on the MObjects exploration and the resulting representative MObjects, the domain experts take the mean pores of a real dataset as input for the heat conduction simulations. As their method

Table 1. Summary of the Evaluation Questionnaire

\begin{tabular}{|c|c|c|}
\hline$\frac{\bar{y}}{\sqrt{a}}$ & $\begin{array}{l}\text { Identification of deviating (not nodular) structures } \\
\text { in a 3D rendering } \\
\text { in a MObject visualization }\end{array}$ & $\begin{array}{l}- \\
\pm\end{array}$ \\
\hline \multirow{2}{*}{$\begin{array}{l}\frac{1}{\mathfrak{m}} \\
\frac{\pi}{\mathfrak{m}}\end{array}$} & $\begin{array}{l}\text { Pore homogeneity identification of the dataset } \\
\text { in the individual pores visualization } \\
\text { in the local MObjects visualization } \\
\text { in the color-coded homogeneity visualization }\end{array}$ & $\begin{array}{l}\mp \\
\pm \\
+\end{array}$ \\
\hline & $\begin{array}{l}\text { Pore homogeneity identification in the cells } \\
\text { in the individual pores visualization } \\
\text { in the local MObjects visualization } \\
\text { in the color-coded homogeneity visualization }\end{array}$ & $\begin{array}{l}- \\
\pm \\
\circ\end{array}$ \\
\hline \multirow{2}{*}{$\begin{array}{l}m \\
\frac{y}{\pi} \\
\frac{\pi}{\pi}\end{array}$} & $\begin{array}{l}\text { Identification of the selected path and the different scal- } \\
\text { ings through visual linking in a parallel MObject Set vi- } \\
\text { sualization }\end{array}$ & + \\
\hline & $\begin{array}{l}\text { Identification of representative pore classifications } \\
\text { in a } 3 \mathrm{D} \text { rendering } \\
\text { in a MObject visualization }\end{array}$ & $\begin{array}{l}\mp \\
+\end{array}$ \\
\hline & - poor, $\mp$ fair, $\circ$ average, \pm very good, + excellent & \\
\hline
\end{tabular}


assumes a homogenous distribution of the pores, the homogeneity visualization of the different pore properties is important (Local MObjects Visualization - Task 2). These new possibilities aim to improve the simulations and finally the active-thermography method. The reference methods ultrasonic testing and active thermography only provide $2 \mathrm{D}$ images with lower resolutions. Due to the missing $3 \mathrm{D}$ information, we were not able to directly compare the MObjects visualization to existing NDT methods. As the common XCT visualization is a $3 \mathrm{D}$ rendering of the dataset, we compare our MObject visualizations to 3D renderings of segmented pores instead. Until now there has not been an averaging approach available to visually explore the characteristics of defects in XCT datasets in 3D.

The questionnaire focuses on the evaluation of the three tasks concerning the MObjects visualization (MObjects Visualization - Task 1), the homogeneity visualization (Local MObjects Visualization - Task 2 ), and the MObjects exploration and extraction of representative MObjects (MObjects Exploration - Task 3) in a dataset. A summary of the evaluation-questionnaire results is shown in Table 1.

MObjects Visualization (Task 1): Regarding the MObject visualization, the experts were shown 3D renderings of individual objects in isometric and $\mathrm{xz}$ views as well as $3 \mathrm{D}$ renderings of the corresponding MObject visualizations. Two different transfer function settings influence the visual depiction of the uncertainty cloud. In the questionnaire the respondents were asked whether they can identify deviating structures in the visualizations. As the used dataset mainly contains nodular pores, apart from a few elongated objects with a different shape factor, the respondents were asked to identify deviating structures like non-nodular outliers. The feedback confirms, that it is easier to find deviating structures in the MObject visualizations than in the 3D renderings showing the individual objects. This is due to the occlusion of the individual objects, so that deviating structures are hardly or not at all seen in a 3D rendering. Considering the answers about the different transfer function settings in the MObject visualizations, a slight trend is recognizable. Although the outliers can be clearly seen in both MObject visualizations ( $\sigma=0.15$ and $\sigma=0.9$ ), due to perceptional reasons the respondents prefer a setting where the uncertainty cloud clearly separates the core from the outliers $(\sigma=0.9)$.

Local MObjects Visualization (Task 2): To evaluate the local MObjects visualization, we compared the visualization of segmented pores in a CFRP dataset, the corresponding visualization of local MObjects in a 4 x 3 grid, and the color-coded homogeneity visualization of the same dataset. The task for the respondents was to find the cells with lowest, highest positive and highest negative deviation of the pore properties in the segmented pores and the color-coded homogeneity visualization. It was nearly impossible for the participants to see the pore homogeneity in the segmented pores visualization whereas they were able to properly classify all cells in the color-coded homogeneity visualization. Regarding the local MObjects visualization the respondents were asked to find the MObjects with lowest and highest average pore properties in the dataset as well as the lowest and highest pore homogeneity in the cells. Generally the respondents judged our local MObjects visualization positively for perceiving the pore homogeneity inside the cells. To convey the pore homogeneity of the whole dataset across all the cells the color-coded homogeneity visualization was rated as a highly helpful visualization. Especially active thermography experts gain new homogeneity information out of our visualization to evaluate and evolve their method.

MObjects Exploration (Task 3): Another part of the questionnaire addressed the interactive MObjects exploration approach. The respondents rated as high the need of the exploration to find nodular, long and thin as well as crack-shaped objects. Furthermore they agreed that the classifications based on the pore volume, the dimensions and the shape factors are very interesting. There is a slight preference to take the shape factors as the most important property. For each property the experts typically split up a MObject into two to five sub-classes. All respondents were able to identify the selected path in a MObject Set visualization. The visual linking approach was positively received, as it allows to see the different scalings of the different MObjects along the selected path. The last aspect of the evaluation comprises the exploration of representative mean pores in a CFRP dataset. The respondents were shown a $3 \mathrm{D}$ rendering of segmented pores in a CFRP specimen and the corresponding MObject visualization. They were hardly able to identify nodular, long and thin micro pores as well as cracks in the rendering of the segmented pores. On the other hand they positively valued the MObject visualization where it was easy for them to identify the different pore classifications. Furthermore we showed the respondents renderings of MObjects with different pore classifications. These were found with the MObjects exploration approach. The respondents agreed that the MObjects convey the typical pore structures in a CFRP dataset.

Further Feedback: NDT practitioners mentioned that the MObjects visualization may have considerable practical relevance in future inline XCT systems for $100 \%$ testing in quality control. An automatic MObject evaluation tool may single out components with critical defects.

\section{Conclusions AND Future Work}

A novel method for the visualization and interactive exploration of MObjects was presented. We calculate MObjects from a set of individual objects and visualize them by applying transfer functions. As the MObjects dataset stores probabilities, the transfer function design is guided by a user-defined uncertainty filter. The approach includes the visualization of pore homogeneities as well. Local MObjects are visualized on a regular grid to show the pore homogeneity in individual cells. To visualize the pore homogeneity of the whole dataset, a colorcoded homogeneity visualization was implemented which shows the deviation from the average pore properties. For the exploration with our MObject Set visualization we proposed a beginners mode in radial arrangement and an expert mode in parallel arrangement. Besides the interactive selection of MObjects, we introduced a scaling through visual linking approach along a selected path. Single MObjects hold probabilities for each voxel of belonging to the MObject and are visualized using transfer functions. They can be exported as volumetric dataset to drive subsequent calculations or simulations.

We have applied our techniques to cases of high practical relevance in the aeronautics industry. Representative pores of a CFRP dataset were found during interactive exploration of the MObjects. Based on these results NDT practitioners calculate the most appropriate ultrasonic calibration curve for each component to be tested in quality control. In active thermography the representative pores serve as input for the heat conduction simulations to improve the underlying model. Domain experts of a company manufacturing aircraft components and active thermography experts evaluated the MObjects approach with a questionnaire and found it to be a helpful tool with high practical relevance.

Although we focused on the evaluation of pores in this work, our approach can be used for all kinds of defects in material sciences. In the future we will apply our methods on cracks, inclusions, particles and fibers. Currently the number of classes into which a parent $\mathrm{MOb}-$ ject is split up regarding a specific property is user-defined. This is based on the knowledge of the domain experts as well as norms specifying the number of partitions for certain properties. For future work the number of classes, a MObject is split up, can be chosen by an automatic approach.

\section{ACKNOWLEDGMENTS}

This project was supported by the program Regionale Wettbewerbsfähigkeit OÖ 2010-2013, which is financed by the European Regional Development Fund and the Government of Upper Austria. Furthermore this work is partly financed by the K-Project ZPT of the Austrian Research Promotion Agency (FFG). The research leading to these results has also received funding from the European Union's Seventh Framework Programme (FP7/2007-2013) under grant agreement ACP2-GA-2012-314562-QUICOM. 


\section{REFERENCES}

[1] J. Ahrens, K. Heitmann, M. Petersen, J. Woodring, S. Williams, P. Fasel, C. Ahrens, C.-H. Hsu, and B. Geveci. Verifying Scientific Simulations via Comparative and Quantitative Visualization. Computer Graphics and Applications, IEEE, 30(6):16-28, 2010.

[2] A. Amirkhanov, C. Heinzl, M. Reiter, and M. E. Gröller. Visual Optimality and Stability Analysis of 3DCT Scan Positions. IEEE Transactions on Visualization and Computer Graphics, pages 1477-1487, October 2010.

[3] C. Bajaj, V. Pascucci, and D. Schikore. The Contour Spectrum. In Proceedings of IEEE Visualization '97, pages 167-173, October 1997.

[4] J.-P. Balabanian, I. Viola, and E. Gröller. Interactive Illustrative Visualization of Hierarchical Volume Data. In Proceedings of Graphics Interface 2010, pages 137-144, June 2010.

[5] W. Berger, H. Piringer, P. Filzmoser, and E. Gröller. Uncertainty-Aware Exploration of Continuous Parameter Spaces Using Multivariate Prediction. Computer Graphics Forum, 30(3):911-920, June 2011.

[6] A. Brambilla, I. Viola, and H. Hauser. A Hierarchical Splitting Scheme to Reveal Insight into Highly Self-Occluded Integral Surfaces. Journal of WSCG, 20(1):5764, July 2012.

[7] S. Bruckner and T. Möller. Result-Driven Exploration of Simulation Parameter Spaces for Visual Effects Design. IEEE Transactions on Visualization and Computer Graphics, 16(6):1467-1475, October 2010.

[8] S. Bruckner, V. Šoltészová, E. Gröller, J. Hladůvka, K. Bühler, J. Yu, and B. Dickson. BrainGazer - Visual Queries for Neurobiology Research. IEEE Transactions on Visualization and Computer Graphics, 15(6): 1497-1504, November 2009.

[9] N. Fout and K.-L. Ma. Fuzzy Volume Rendering. IEEE Transactions on Visualization and Computer Graphics, 18(12):2335-2344, December 2012.

[10] G. Grigoryan and P. Rheingans. Point-Based Probabilistic Surfaces to Show Surface Uncertainty. IEEE Transactions on Visualization and Computer Graphics, 10(5):564-573, September 2004.

[11] C. Heinzl, J. Kastner, T. Möller, and E. Gröller. Statistical Analysis of Multi-Material Components using Dual Energy CT. In VMV 2008, Vision, Modeling and Visualization, pages 179-188, October 2008.

[12] A. Inselberg. Parallel Coordinates: Visual Multidimensional Geometry and Its Applications. Springer-Verlag New York, Inc., Secaucus, NJ, USA, 2009.

[13] C. Y. Ip, A. Varshney, and J. F. Jaja. Hierarchical Exploration of Volumes Using Multilevel Segmentation of the Intensity-Gradient Histograms. IEEE Transactions on Visualization and Computer Graphics, 18(12):2355-2363, December 2012.

[14] J. Kniss, R. Van Uitert, A. Stephens, G.-S. Li, T. Tasdizen, and C. Hansen. Statistically quantitative volume visualization. In Proceedings of IEEE Visualization 2005, pages 287 - 294, oct. 2005

[15] R. Kosara, F. Bendix, and H. Hauser. Parallel Sets: Interactive Exploration and Visual Analysis of Categorical Data. IEEE Transactions on Visualization and Computer Graphics, 12(4):558-568, July/August 2006.

[16] A. Lex, M. Streit, H.-J. Schulz, C. Partl, D. Schmalstieg, P. J. Park, and N. Gehlenborg. StratomeX: Visual Analysis of Large-Scale Heterogeneous Genomics Data for Cancer Subtype Characterization. Computer Graphics Forum, 31(3):1175-1184, 2012.

[17] K.-L. Ma. Image Graphs - A Novel Approach to Visual Data Exploration. In Proceedings of IEEE Visualization '99, pages 81-88, 1999.

[18] M. Malik, C. Heinzl, and E. Gröller. Comparative Visualization for Parameter Studies of Dataset Series. IEEE Transactions on Visualization and Computer Graphics, 16(5):829-840, September/October 2010.

[19] J. Marks, B. Andalman, P. A. Beardsley, W. Freeman, S. Gibson, J. Hodgins, T. Kang, B. Mirtich, H. Pfister, W. Ruml, K. Ryall, J. Seims, and S. Shieber. Design galleries: A General Approach to Setting Parameters for Computer Graphics and Animation. In Proceedings of the 24th annual conference on Computer graphics and interactive techniques, SIGGRAPH '97, pages 389-400, New York, NY, USA, 1997. ACM Press/Addison-Wesley Publishing Co.

[20] G. Mayr, B. Plank, J. Sekelja, and G. Hendorfer. Active thermography as a quantitative method for non-destructive evaluation of porous carbon fiber reinforced polymers. NDT \& E International, 44(7):537-543, 2011.

[21] N. Otsu. A Threshold Selection Method from Gray-Level Histograms. IEEE Trans. Systems, Man and Cybernetics, 9:62-66, March 1979.

[22] P. Perona and J. Malik. Scale-Space and Edge Detection Using Anisotropic Diffusion. IEEE Trans. Pattern Anal. Mach. Intell., 12:629639, July 1990.
[23] A. Reh, B. Plank, J. Kastner, E. Gröller, and C. Heinzl. Porosity Maps Interactive Exploration and Visual Analysis of Porosity in Carbon Fiber Reinforced Polymers. Computer Graphics Forum, 31(3):1185-1194, June 2012.

[24] M. Sezgin and B. Sankur. Survey over image thresholding techniques and quantitative performance evaluation. J. Electronic Imaging, 13(1):146$168,2004$.

[25] C. Soutis. Fibre reinforced composites in aircraft construction. Progress in Aerospace Sciences, 41(2):143-151, February 2005.

[26] T. Torsney-Weir, A. Saad, T. Moller, H. Hege, B. Weber, J. Verbavatz, and S. Bergner. Tuner: Principled Parameter Finding for Image Segmentation Algorithms Using Visual Response Surface Exploration. IEEE Transactions on Visualization and Computer Graphics, 17(12):1892-1901, December 2011

[27] J. J. van Wijk and R. van Liere. HyperSlice: Visualization of scalar functions of many variables. In Proceedings of IEEE Visualization '93, pages 119-125, October 1993.

[28] J. Woodring and H.-W. Shen. Multiscale Time Activity Data Exploration via Temporal Clustering Visualization Spreadsheet. IEEE Transactions on Visualization and Computer Graphics, 15(1):123-137, January/February 2009.

[29] R. Xu. Survey of Clustering Algorithms. IEEE Transactions on Neural Networks, 16(3):645-678, May 2005. 